\title{
Model independent constraints on mass-varying neutrino scenarios
}

\author{
Urbano França, ${ }^{1}$ Massimiliano Lattanzi, ${ }^{2}$ Julien Lesgourgues, ${ }^{3}$ and Sergio Pastor ${ }^{1}$ \\ ${ }^{1}$ Instituto de Física Corpuscular (CSIC-Universitat de València), Edificio Institutos de Investigación, Apartado 22085,46071 \\ Valencia, Spain \\ ${ }^{2}$ ICRA Dipartimento di Fisica, Universitá di Roma “La Sapienza," Piazzale Aldo Moro 2, 00185 Roma, Italy \\ ${ }^{3}$ CERN, Theory Division, CH-1211 Geneva 23, Switzerland, Institut de Théorie des Phénomènes Physiques, \\ EPFL, CH-1015 Lausanne, Switzerland, \\ and LAPTH (CNRS-Universié de Savoie), B.P. 110, F-74941 Annecy-le-Vieux Cedex, France
}

(Received 11 August 2009; published 7 October 2009)

\begin{abstract}
Models of dark energy in which neutrinos interact with the scalar field supposed to be responsible for the acceleration of the Universe usually imply a variation of the neutrino masses on cosmological time scales. In this work we propose a parametrization for the neutrino mass variation that captures the essentials of those scenarios and allows one to constrain them in a model independent way, that is, without resorting to any particular scalar field model. Using WMAP $5 \mathrm{yr}$ data combined with the matter power spectrum of SDSS and 2dFGRS, the limit on the present value of the neutrino mass is $m_{0} \equiv m_{\nu}(z=$ $0)<0.43(0.28) \mathrm{eV}$ at $95 \%$ C.L. for the case in which the neutrino mass was lighter (heavier) in the past, a result competitive with the ones imposed for standard (i.e., constant mass) neutrinos. Moreover, for the ratio of the mass variation of the neutrino mass $\Delta m_{\nu}$ over the current mass $m_{0}$ we found that $\log \left[\left|\Delta m_{\nu}\right| / m_{0}\right]<-1.3(-2.7)$ at $95 \%$ C.L. for $\Delta m_{\nu}<0\left(\Delta m_{\nu}>0\right)$, totally consistent with no mass variation. These stringent bounds on the mass variation are not related to the neutrino freestreaming history which may affect the matter power spectrum on small scales. On the contrary, they are imposed by the fact that any significant transfer of energy between the neutrino and dark energy components would lead to an instability contradicting CMB and large-scale structure data on the largest observable scales.
\end{abstract}

DOI: $10.1103 /$ PhysRevD.80.083506

PACS numbers: 98.80.-k, 14.60.St, 98.80.Cq, 98.80.Es

\section{INTRODUCTION}

Since the accelerated expansion of the Universe was first observed with type Ia supernovae (SN) [1,2], the case for a cosmological constantlike fluid that dominates the energy density of the Universe has become stronger and is well established by now with the new pieces of data gathered [3].

Several candidates for the accelerating component of the Universe, generically dubbed dark energy (DE), have been proposed [3-6], but understanding them theoretically and observationally has proven to be challenging. On the theoretical side, explaining the small value of the observed dark energy density component, $\rho_{\phi} \sim\left(10^{-3} \mathrm{eV}\right)^{4}$, as well as the fact that both dark energy and matter densities contribute significantly to the energy budget of the present Universe requires in general a strong fine-tuning on the overall scale of the dark energy models. In the case in which the dark energy is assumed to be a scalar field $\phi$ slowly rolling down its flat potential $V(\phi)$, the so-called quintessence models [7], the effective mass of the field has to be taken of the order $m_{\phi}=\left|d^{2} V(\phi) / d \phi^{2}\right|^{1 / 2} \sim$ $10^{-33} \mathrm{eV}$ for fields with vacuum expectation values of the order of the Planck mass.

On the observational side, choosing among the dark energy models is a complicated task [8]. Most of them can mimic a cosmological constant at late times (that is, an equation of state $w_{\phi} \equiv p_{\phi} / \rho_{\phi}=-1$ ) [9], and all data until now are perfectly consistent with this limit. In this sense, looking for different imprints that could favor the existence of a particular model of dark energy is a path worth taking.

Our goal in this paper consists in understanding whether the so-called mass-varying neutrinos (MaVaNs) scenario [10-14] could be constrained not only via the dark energy effects, but also by indirect signs of the neutrino mass variation during cosmological evolution, since neutrinos play a key role in several epochs $[15,16]$. An indication of the variation of the neutrino mass would certainly tend to favor these models (at least on a theoretical basis) with respect to most DE models. One should keep in mind that MaVaNs scenarios can suffer from stability issues for the neutrino perturbations [17], although there is a wide class of models and couplings that avoid this problem [18-22].

Similar analyses have been made in the past, but they have either assumed particular models for the interaction between the neutrinos and the DE field [23-25], or chosen a parametrization that does not reflect the richness of the possible behavior of the neutrino mass variations [26].

In order to be able to deal with a large number of models, instead of focusing on a particular model for the coupling between the DE field and the neutrino sector, we choose to parametrize the neutrino mass variation to place general and robust constraints on the MaVaNs scenario. In this sense, our work complements previous analyses by assuming a realistic and generic parametrization for the neutrino 
mass, designed in such a way to probe almost all the different regimes and models within the same framework. In particular, our parametrization allows for fast and slow mass transitions between two values of the neutrino mass, and it takes into account that the neutrino mass variation should start when the coupled neutrinos change their behavior from relativistic to nonrelativistic species. We can mimic different neutrino-dark energy couplings and allow for almost any monotonic behavior in the neutrino mass, placing reliable constraints on this scenario in a model independent way.

Our work is organized as follows: in Sec. II we give a brief review of the MaVaNs scenario and its main equations. In Sec. III we present our parametrization with the results for the background and the perturbation equations obtained within this context. The results of our comparison of the numerical results with the data and the discussion of its main implications are shown in Sec. IV. Finally, in Sec. V the main conclusions and possible future directions are discussed.

\section{MASS-VARYING NEUTRINOS}

In what follows, we consider a homogeneous and isotropic universe with a Robertson-Walker flat metric, $d s^{2}=$ $a^{2}\left(d \tau^{2}+d r^{2}+r^{2} d \Omega^{2}\right)$, where $\tau$ is the conformal time that can be written in terms of the cosmic time $t$ and scale factor $a$ as $d \tau=d t / a$, in natural units $\left(\hbar=c=k_{B}=1\right)$. In this case, the Friedmann equations read

$$
\begin{aligned}
& \mathcal{H}^{2}=\left(\frac{\dot{a}}{a}\right)^{2}=\frac{a^{2}}{3 m_{p}^{2}} \rho, \\
& \mathcal{H}=-\frac{a^{2}}{6 m_{p}^{2}}(\rho+3 p),
\end{aligned}
$$

where the dot denotes a derivative with respect to conformal time, and the reduced Planck mass is $m_{p}=$ $1 / \sqrt{8 \pi G}=2.436 \times 10^{18} \mathrm{GeV}$. As usual, $\rho$ and $p$ correspond to the total energy density and pressure of the cosmic fluid, respectively. The neutrino mass in the models we are interested in is a function of the scalar field $\phi$ that plays the role of the dark energy, and can be written as

$$
m_{\nu}(\phi)=M_{\nu} f(\phi),
$$

where $M_{\nu}$ is a constant and different models are represented by distinct $f(\phi)$.

The fluid equation of the neutrino species can be directly obtained from the Boltzmann equation for its distribution function [24],

$$
\dot{\rho}_{\nu}+3 \mathcal{H} \rho_{\nu}\left(1+w_{\nu}\right)=\alpha(\phi) \dot{\phi}\left(\rho_{\nu}-3 p_{\nu}\right),
$$

where $\alpha(\phi)=d \ln \left[m_{\nu}(\phi)\right] / d \phi$ takes into account the variation of the neutrino mass, and $w_{x}=p_{x} / \rho_{x}$ is the equation of state of the species $x$. For completeness and later use, we will define $\Omega_{x 0}=\rho_{x} / \rho_{c 0}$, the standard density parameter, where the current critical density is given by $\rho_{c 0}=3 H_{0}^{2} m_{p}^{2}=8.099 h^{2} \times 10^{-11} \mathrm{eV}^{4}$, and $H_{0}=$ $100 \mathrm{hm} \mathrm{s}^{-1} \mathrm{Mpc}^{-1}$ is the Hubble constant.

Since the total energy momentum tensor is conserved, the dark energy fluid equation also presents an extra righthand side term proportional to the neutrino energy momentum tensor trace, $T_{(\nu) \alpha}^{\alpha}=\left(\rho_{\nu}-3 p_{\nu}\right)$, and can be written as

$$
\dot{\rho}_{\phi}+3 \mathcal{H} \rho_{\phi}\left(1+w_{\phi}\right)=-\alpha(\phi) \dot{\phi}\left(\rho_{\nu}-3 p_{\nu}\right) .
$$

For a homogeneous and isotropic scalar field, the energy density and pressure are given by

$$
\rho_{\phi}=\frac{\dot{\phi}^{2}}{2 a^{2}}+V(\phi), \quad p_{\phi}=\frac{\dot{\phi}^{2}}{2 a^{2}}-V(\phi),
$$

and both equations lead to the standard cosmological Klein-Gordon equation for an interacting scalar field, namely,

$$
\ddot{\phi}+2 \mathcal{H} \dot{\phi}+a^{2} \frac{d V(\phi)}{d \phi}=-a^{2} \alpha(\phi)\left(\rho_{\nu}-3 p_{\nu}\right) .
$$

From the above equations one sees that, given a potential $V(\phi)$ for the scalar field and a field-dependent mass term $m_{\nu}(\phi)$ for the neutrino mass, the coupled system given by Eqs. (1), (4), and (7), together with the fluid equations for the baryonic matter, cold dark matter and radiation (photons and other massless species) can be numerically solved [24]. Notice that a similar approach has been used for a possible variation of the dark matter mass [27] and its possible interaction with the dark energy $[28,29]$, with several interesting phenomenological ramifications [3035].

Following [31,33], Eqs. (4) and (5) can be rewritten in the standard form,

$$
\begin{gathered}
\dot{\rho}_{\nu}+3 \mathcal{H} \rho_{\nu}\left(1+w_{\nu}^{(\text {eff })}=0,\right. \\
\dot{\rho}_{\phi}+3 \mathcal{H} \rho_{\phi}\left(1+w_{\phi}^{(\text {eff })}=0,\right.
\end{gathered}
$$

if one defines the effective equation of state of neutrinos and $\mathrm{DE}$ as

$$
\begin{aligned}
& w_{\nu}^{(\mathrm{eff})}=\frac{p_{\nu}}{\rho_{\nu}}-\frac{\alpha(\phi) \dot{\phi}\left(\rho_{\nu}-3 p_{\nu}\right)}{3 \mathcal{H} \rho_{\nu}}, \\
& w_{\phi}^{(\mathrm{eff})}=\frac{p_{\phi}}{\rho_{\phi}}+\frac{\alpha(\phi) \dot{\phi}\left(\rho_{\nu}-3 p_{\nu}\right)}{3 \mathcal{H} \rho_{\phi}} .
\end{aligned}
$$

The effective equation of state can be understood in terms of the dilution of the energy density of the species. In the standard noncoupled case, the energy density of a fluid with a given constant equation of state $w$ scales as $\rho \propto$ $a^{-3(1+w)}$. However, in the case of interacting fluids, one should also take into account the energy transfer between them, and the energy density in this case will be given by

$$
\rho(z)=\rho_{0} \exp \left[3 \int_{0}^{z}\left(1+w^{(\mathrm{eff})}\left(z^{\prime}\right)\right) d \ln \left(1+z^{\prime}\right)\right],
$$


where the index 0 denotes the current value of a parameter, and the redshift $z$ is defined by the expansion of the scale factor, $a=a_{0}(1+z)^{-1}$ (in the rest of this work we will assume $a_{0}=1$ ). For a constant effective equation of state one obtains the standard result, $\rho \propto a^{-3\left(1+w^{(\text {eff })}\right)}$, as expected.

Notice that this mismatch between the effective and standard DE equations of state could be responsible for the "phantom behavior" suggested by supernovae data when fitting it using a cosmological model with noninteracting components [33]. This effect could be observable if dark energy was coupled to the dominant dark matter component. For the models discussed here, however, it cannot be significant: the neutrino fraction today $\left(\Omega_{\nu 0} / \Omega_{\phi 0} \sim 10^{-2}\right)$ is too small to induce an "effective phantomlike" behavior.

As we commented before, the analysis until now dealt mainly with particular models, that is, with particular functional forms of the dark energy potential $V(\phi)$ and field dependence of the neutrino mass $\alpha(\phi)$. A noticeable exception is the analysis of Ref. [26], in which the authors use a parametrization for the neutrino mass à la ChevallierPolarski-Linder $\quad(\mathrm{CPL}) \quad[9,36,37]: \quad m_{\nu}(a)=m_{\nu 0}+$ $m_{\nu 1}(1-a)$. However, although the CPL parametrization works well for the dark energy equation of state, it cannot reproduce the main features of the mass variation in the case of variable mass particle models. In the case of the models discussed here, for instance, the mass variation is related to the relativistic/nonrelativistic nature of the coupled neutrino species. With a CPL mass parametrization, the transition from $m_{1}$ to $m_{0}$ always takes place around $z \sim 1$, which is in fact only compatible with masses as small as $10^{-3} \mathrm{eV}$. Hence, the CPL mass parametrization is not suited for a self-consistent exploration of all interesting possibilities.

One of the goals in this paper is to propose and test a parametrization that allows for a realistic simulation of mass-varying scenarios in a model independent way, with the minimum possible number of parameters, as explained in the next section.

\section{MODEL INDEPENDENT APPROACH}

\section{A. Background equations}

As usual, the neutrino energy density and pressure are given in terms of the zero order Fermi-Dirac distribution function by

$$
f^{0}(q)=\frac{g_{\nu}}{e^{q / T_{\nu 0}}+1},
$$

where $q=a p$ denotes the modulus of the comoving momentum $q_{i}=q n_{i}\left(\delta^{i j} n_{i} n_{j}=1\right), g_{\nu}$ corresponds to the number of neutrino degrees of freedom, and $T_{\nu 0}$ is the present neutrino background temperature. Notice that in the neutrino distribution function we have used the fact that the neutrinos decouple very early in the history of the
Universe while they are relativistic, and therefore their equilibrium distribution depends on the comoving momentum, but not on the mass [16]. In what follows we have neglected the small spectral distortions arising from noninstantaneous neutrino decoupling [38]. Thus, the neutrino energy density and pressure are given by

$$
\begin{gathered}
\rho_{\nu}=\frac{1}{a^{4}} \int \frac{d q}{(2 \pi)^{3}} d \Omega q^{2} \epsilon f^{0}(q), \\
p_{\nu}=\frac{1}{3 a^{4}} \int \frac{d q}{(2 \pi)^{3}} d \Omega q^{2} f^{0}(q) \frac{q^{2}}{\epsilon},
\end{gathered}
$$

where $\epsilon^{2}=q^{2}+m_{\nu}^{2}(a) a^{2}$ (assuming that $m_{\nu}$ depends only on the scale factor). Taking the time derivative of the energy density, one can then obtain the fluid equation for the neutrinos,

$$
\dot{\rho}_{\nu}+3 \mathcal{H}\left(\rho_{\nu}+p_{\nu}\right)=\frac{d \ln m_{\nu}(u)}{d u} \mathcal{H}\left(\rho_{\nu}-3 p_{\nu}\right),
$$

where $u \equiv \ln a=-\ln (1+z)$ is the number of e-folds counted back from today. Because of the conservation of the total energy momentum tensor, the dark energy fluid equation is then given by

$$
\dot{\rho}_{\phi}+3 \mathcal{H} \rho_{\phi}\left(1+w_{\phi}\right)=-\frac{d \ln m_{\nu}(u)}{d u} \mathcal{H}\left(\rho_{\nu}-3 p_{\nu}\right) .
$$

We can write the effective equations of state, defined in Eqs. (8), as

$$
\begin{aligned}
& w_{\nu}^{\mathrm{eff}}=\frac{p_{\nu}}{\rho_{\nu}}-\frac{d \ln m_{\nu}(u)}{d u}\left(\frac{1}{3}-\frac{p_{\nu}}{\rho_{\nu}}\right), \\
& w_{\phi}^{\mathrm{eff}}=\frac{p_{\phi}}{\rho_{\phi}}+\left(\frac{\Omega_{\nu}}{\Omega_{\phi}}\right) \frac{d \ln m_{\nu}(u)}{d u}\left(\frac{1}{3}-\frac{p_{\nu}}{\rho_{\nu}}\right) .
\end{aligned}
$$

The above results only assume that the neutrino mass depends on the scale factor $a$, and up to this point, we have not chosen any particular parametrization. Concerning the particle physics models, it is important to notice that starting from a value of $w_{\phi}$ and a function $m_{\nu}(a)$ one could, at least in principle, reconstruct the scalar potential and the scalar interaction with neutrinos following an approach similar to the one in Ref. [39].

\section{B. Mass variation parameters}

Some of the main features of the MaVaNs scenario are (i) that the dark energy field gets kicked and moves away from its minimum (if $m_{\phi}>H$ ) or from its previous slowrolling trajectory (if $m_{\phi}<H$ ) when the neutrinos become nonrelativistic, very much like the case when it is coupled to the full matter content of the Universe in the so-called chameleon scenarios [40]; and (ii) that as a consequence, the coupling with the scalar field generates a neutrino mass variation at that time. Any parametrization that intends to mimic scalar field models interacting with a mass-varying particle (neutrinos, in our case) for the large redshift range 
to which the data are sensitive should at least take into account those characteristics. Moreover, the variation of the mass in most models (see [24], for instance) can be well approximated by a transition between two periods: an earlier one, in which the mass is given by $m_{1}$, and the present epoch, in which the mass is given by $m_{0}$ (we will not consider here models in which the neutrino mass behavior is nonmonotonic). The transition for this parametrization, as mentioned before, starts when neutrinos become nonrelativistic, which corresponds approximately to

$$
z_{\mathrm{NR}} \approx 1.40\left(\frac{1 \mathrm{eV}}{3 T_{\gamma 0}}\right)\left(\frac{m_{1}}{1 \mathrm{eV}}\right) \approx 2 \times 10^{3}\left(\frac{m_{1}}{1 \mathrm{eV}}\right),
$$

where $m_{1}$ corresponds to the mass of the neutrino during the period in which it is a relativistic species. Before $z_{\mathrm{NR}}$ we can treat the neutrino mass as essentially constant, since the right-hand side (rhs) of the fluid equation is negligible compared to the left-hand side (lhs), and therefore there is no observable signature of a possible mass variation.

When the neutrinos become nonrelativistic, the rhs of the DE and neutrino fluid equations becomes important, and the neutrino mass starts varying. In order to model this variation, we use two parameters, namely, the current neutrino mass, $m_{0}$, and $\Delta$, a quantity related to the amount of time that it takes to complete the transition from $m_{1}$ to $m_{0}$. That behavior resembles very much the parametrization of the dark energy equation of state discussed in [41], except for the fact that in our case the transition for the mass can be very slow, taking several e-folds to complete, and must be triggered by the time of the nonrelativistic transition, given by Eq. (17). Defining $f=[1+$ $\left.e^{-\left[u(1+\Delta)-u_{\mathrm{NR}}\right] / \Delta}\right]^{-1}$ and $f_{*}=\left[1+e^{u_{\mathrm{NR}} / \Delta}\right]^{-1}$ we can use the form

$$
m_{\nu}=m_{0}+\left(m_{1}-m_{0}\right) \times \Gamma\left(u, u_{\mathrm{NR}}, \Delta\right),
$$

where

$$
\Gamma\left(u, u_{\mathrm{NR}}, \Delta\right)=1-\frac{f}{f_{*}}=\left[1-\frac{1+e^{u_{\mathrm{NR}} / \Delta}}{1+e^{-\left[u(1+\Delta)-u_{\mathrm{NR}}\right] / \Delta}}\right] .
$$

Starting at $u_{\mathrm{NR}}=-\ln \left(1+z_{\mathrm{NR}}\right)$, the function $\Gamma\left(u, u_{\mathrm{NR}}, \Delta\right)$ decreases from 1 to 0 , with a velocity that depends on $\Delta$. The top panel in Fig. 1 gives the behavior of Eq. (18) with different parameters; the bottom panel shows that in this parametrization, the derivative of the mass with respect to the e-fold number resembles a Gaussian function. The peak of the quantity $d m / d u$ occurs at the value $\bar{u}=u_{\mathrm{NR}} /(1+$ $\Delta)$; hence, for $\Delta \ll 1$, the mass variation takes place immediately after the nonrelativistic transition $\left(\bar{u} \simeq u_{\mathrm{NR}}\right)$ and lasts a fraction of e-folds (roughly, $3 \Delta$ e-folds). For $1 \leq \Delta \leq\left|u_{\mathrm{NR}}\right|$ the variation is smooth and centered on some intermediate redshift between $z_{\mathrm{NR}}$ and 0 , while for $\Delta \gg\left|u_{\mathrm{NR}}\right|$, the transition is still ongoing today, and the present epoch roughly coincides with the maximum variation.
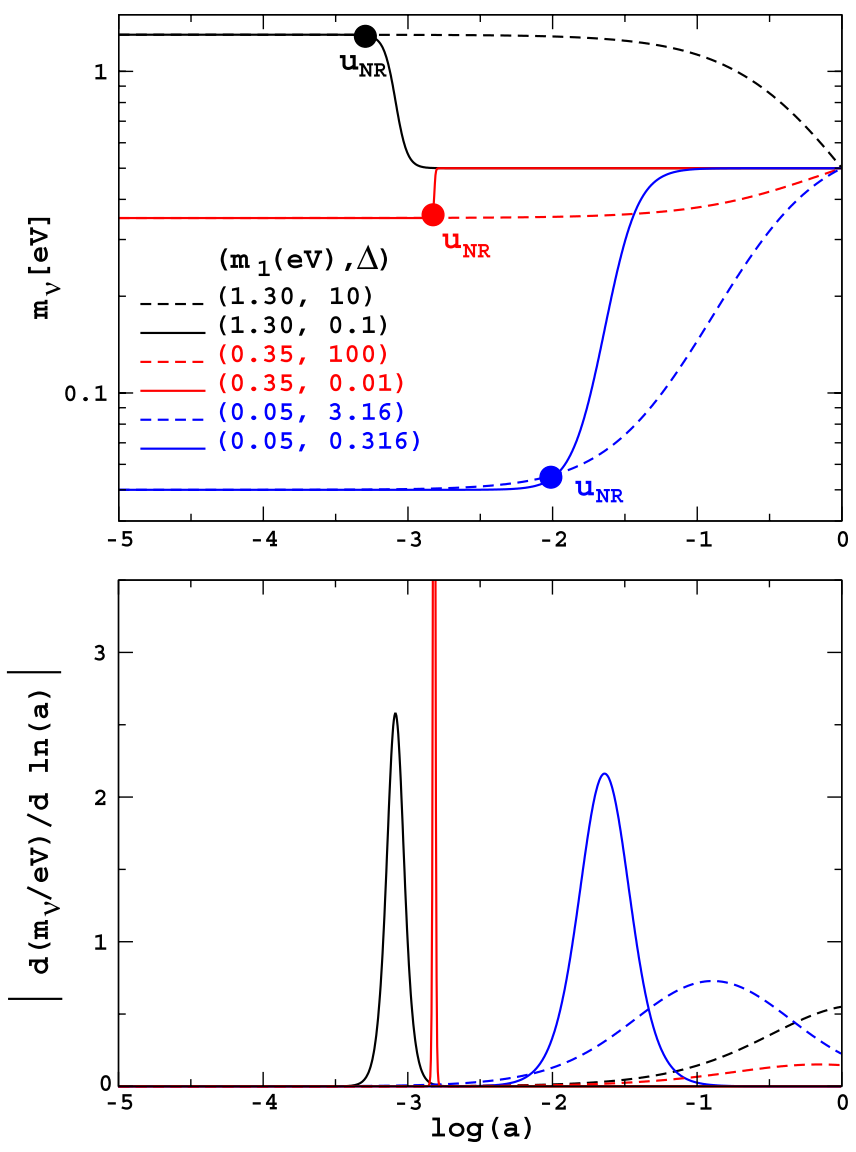

FIG. 1 (color online). Neutrino mass behavior for the parametrization given by Eq. (18). Top panel: Neutrino mass as a function of $\log (a)=u / \ln (10)$ for models with $m_{0}=0.5 \mathrm{eV}$ and different values of $m_{1}$ and $\Delta$. Bottom panel: Neutrino mass variation for the same parameters as in the top panel.

Although the functional form of $\Gamma$, Eq. (19), seems complicated, one should note that it is one of the simplest forms satisfying our requirements with a minimal number of parameters. An example that could look simpler, but that for practical purposes is not, would be to assume that the two plateaus are linked together by a straight line. In this case, we would need a parametrization of the form

$$
m_{\nu}= \begin{cases}m_{1}, & u<u_{\mathrm{NR}}, \\ m_{0}+\left(m_{1}-m_{0}\right)\left[\frac{u-u_{\mathrm{end}}}{u_{\mathrm{NR}}-u_{\mathrm{end}}},\right. & u_{\mathrm{NR}} \leq u \leq u_{\mathrm{end}}, \\ m_{0}, & u>u_{\mathrm{end}},\end{cases}
$$

where $u_{\text {end }}$ corresponds to the chosen redshift in which the transition stops. Notice that in this case, we not only still have three parameters to describe the mass variation, but also the function is not smooth. Moreover, the derivative of the mass with respect to $u$ gives a top-hat-like function which is discontinuous at both $u_{\mathrm{NR}}$ and $u_{\text {end }}$. In this sense, it seemed to us that Eq. (18) would give us the best "priceto-earnings ratio" among the possibilities to use phenomenologically motivated parametrizations for the massvarying neutrinos, although certainly there could be similar proposals equally viable, such as for instance the possibil- 
ity of adapting for the mass variation the parametrization used for the dark energy equation of state in $[42,43]$. There, the transition between two constant values of the equation of state exhibits a $\tanh \left[\Gamma_{t}\left(u-u_{t}\right)\right]$ dependence, where $\Gamma_{t}$ is responsible for the duration of the transition and $u_{t}$ is related to its halfway point.

In the rest of our analysis, we will use a couple of extra assumptions that need to be taken into account when going through our results. First, we will consider that only one of the three neutrino species is interacting with the dark energy field; that is, only one of the mass eigenstates has a variable mass. The reason for this approximation is twofold: it is a simpler case (compared to the case with 3 varying-mass neutrinos), since instead of 6 extra parameters with respect to the case of constant mass, we have only 2 , namely, the early mass of the neutrino whose mass is varying, $m_{1}$, and the velocity of the transition, related to $\Delta$.

Besides simplicity, the current choice is the only one allowed presently in the case in which neutrinos were heavier in the past. Indeed, we expect our stronger constraints to come from those scenarios, especially if the neutrino species behaves as a nonrelativistic component at the time of radiation-matter equality, given by $1+z_{\mathrm{eq}} \sim$ $4.05 \times 10^{4}\left(\Omega_{c 0} h^{2}+\Omega_{b 0} h^{2}\right) /\left(1+0.23 N_{\text {eff }}\right)$ (here the indices $c$ and $b$ stand for cold dark matter and baryons, respectively, and $N_{\text {eff }}$ is the effective number of relativistic neutrinos). Taking the three neutrino species to be nonrelativistic at equality would change significantly the value of $z_{\text {eq }}$, contradicting CMB data (according to WMAP5, $1+z_{\mathrm{eq}}=3141_{-157}^{+154}$ (68\% C.L.) [44]). Instead, a single neutrino species is still marginally allowed to be nonrelativistic at that time.

To simplify the analysis, we also assumed that the dark energy field, when not interacting with the neutrinos, reached already the so-called scaling solution (see, e.g., [4] and references therein), i.e., the dark energy equation of state $w_{\phi}$ in Eq. (15) is constant in the absence of interaction. Notice however that when the neutrinos become nonrelativistic the dark energy fluid receives the analogous of the chameleon kicks we mentioned before, and the dark energy effective equation of state, Eq. (16), does vary for this period in a consistent way.

The upper panel of Fig. 2 shows how the density parameters of the different components of the Universe evolve in time, in a typical MaVaNs model. The lower panel displays a comparison between mass-varying and constant mass models, in particular, during the transition from $m_{1}$ to $m_{0}$. As one would expect, far from the time of the transition, the densities evolve as they would do in the constant mass case.

\section{Perturbation equations}

The next step is to calculate the cosmological perturbation equations and their evolution using this parametrization. We chose to work in the synchronous gauge, and our
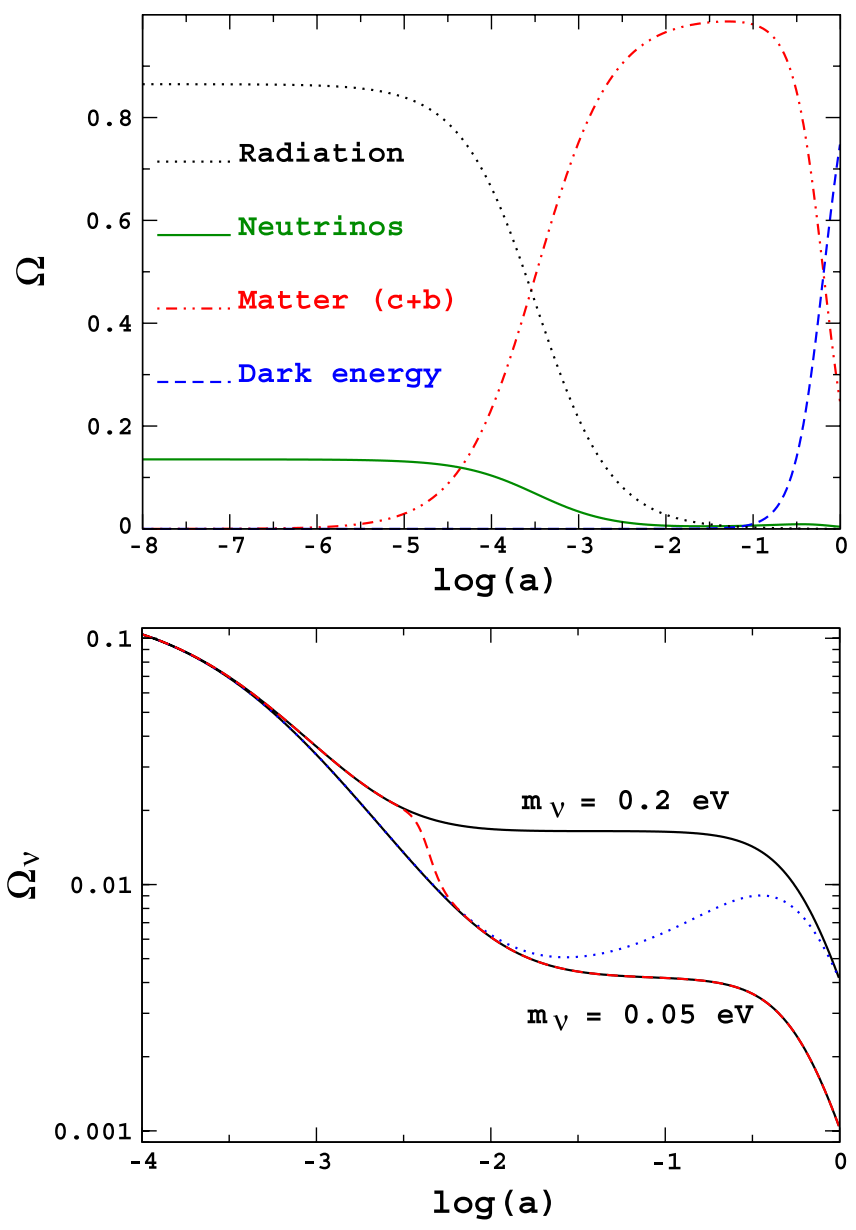

FIG. 2 (color online). Top panel: Density parameters for the different components of the Universe versus $\log (a)=u / \ln (10)$ in a model with $m_{1}=0.05 \mathrm{eV}, m_{0}=0.2 \mathrm{eV}, \Delta=10$, and all the other parameters consistent with the present data. The radiation curve include photons and two massless neutrino species, and matter stands for cold dark matter and baryons. The bump in the neutrino density close to $\log (a)=-0.5$ is due to the increasing neutrino mass. Bottom panel: Density parameters for two different mass-varying neutrino models. The solid black curves show the density parameter variation for two distinct constant mass models, with masses $m_{\nu}=0.05 \mathrm{eV}$ and $m_{\nu}=0.2 \mathrm{eV}$. The dashed (red) curve shows a model in which the mass varies from $m_{1}=0.2 \mathrm{eV}$ to $m_{0}=0.05 \mathrm{eV}$, with $\Delta=$ 0.1 , and the dotted (blue) line corresponds to a model with $m_{1}=$ $0.05 \mathrm{eV}$ to $m_{0}=0.2 \mathrm{eV}$, with $\Delta=10$.

conventions follow the ones by Ma and Bertschinger [45]. In this case, the perturbed metric is given by

$$
d s^{2}=-a^{2} d \tau^{2}+a^{2}\left(\delta_{i j}+h_{i j}\right) d x^{i} d x^{j} .
$$

In this gauge, the equation for the three-momentum of the neutrinos reads [25]

$$
\frac{d q}{d \tau}=-\frac{1}{2} q \dot{h}_{i j} n_{i} n_{j}-a^{2} \frac{m_{v}^{2}}{q} \beta \frac{\partial \rho_{\phi}}{\partial x^{i}} \frac{\partial x^{i}}{d \tau},
$$

where, as in Eq. (4), we define 


$$
\beta(a) \equiv \frac{d \ln m_{\nu}}{d \rho_{\phi}}=\frac{d \ln m_{\nu}}{d \ln a}\left(\frac{d \rho_{\phi}}{d \ln a}\right)^{-1} .
$$

Since the neutrino phase space distribution [45] can be written as $f\left(x^{i}, q, n_{j}, \tau\right)=f^{0}(q)\left[1+\Psi\left(x^{i}, q, n_{j}, \tau\right)\right]$, one can show that the first order Boltzmann equation for a massive neutrino species, after Fourier transformation, is given by $[24,25]$

$$
\begin{aligned}
\frac{\partial \Psi}{\partial \tau} & +i \frac{q}{\epsilon}(\hat{\mathbf{n}} \cdot \mathbf{k}) \Psi+\left(\dot{\eta}-(\hat{\mathbf{k}} \cdot \hat{\mathbf{n}})^{2} \frac{\dot{h}+6 \dot{\eta}}{2}\right) \frac{d \ln f^{0}}{d \ln q} \\
= & -i \beta \frac{q k}{\epsilon}(\hat{\mathbf{n}} \cdot \mathbf{k}) \frac{a^{2} m_{\nu}^{2}}{q^{2}} \frac{d \ln f^{0}}{d \ln q} \delta \rho_{\phi},
\end{aligned}
$$

where $\eta$ and $h$ are the synchronous potentials in the Fourier space. Notice that the perturbed neutrino energy density and pressure are also going to be modified due to the interaction, and are written as

$$
\begin{gathered}
\delta \rho_{\nu}=\frac{1}{a^{4}} \int \frac{d^{3} q}{(2 \pi)^{3}} f^{0}\left(\epsilon \Psi+\beta \frac{m_{\nu}^{2} a^{2}}{\epsilon} \delta \rho_{\phi}\right), \\
3 \delta p_{\nu}=\frac{1}{a^{4}} \int \frac{d^{3} q}{(2 \pi)^{3}} f^{0}\left(\frac{q^{2}}{\epsilon} \Psi-\beta \frac{q^{2} m_{\nu}^{2} a^{2}}{\epsilon^{3}} \delta \rho_{\phi}\right) .
\end{gathered}
$$

This extra term comes from the fact that the comoving energy $\epsilon$ depends on the dark energy density, leading to an extra term which is proportional to $\beta$.

Moreover, if we expand the perturbation $\Psi(\mathbf{k}, q, \mathbf{n}, \tau)$ in a Legendre series [45], the neutrino hierarchy equations will read

$$
\begin{aligned}
& \dot{\Psi}_{0}=-\frac{q k}{\epsilon} \Psi_{1}+\frac{\dot{h}}{6} \frac{d \ln f^{0}}{d \ln q}, \\
& \dot{\Psi}_{1}=\frac{q k}{3 \epsilon}\left(\Psi_{0}-2 \Psi_{2}\right)+\kappa, \\
& \dot{\Psi}_{2}=\frac{q k}{5 \epsilon}\left(2 \Psi_{1}-3 \Psi_{3}\right)-\left(\frac{1}{15} \dot{h}+\frac{2}{5} \dot{\eta}\right) \frac{d \ln f^{0}}{d \ln q}, \\
& \dot{\Psi}_{\ell}=\frac{q k}{(2 \ell+1) \epsilon}\left[\ell \Psi_{\ell-1}-(\ell+1) \Psi_{\ell+1}\right],
\end{aligned}
$$

where

$$
\kappa=-\frac{1}{3} \beta \frac{q k}{\epsilon} \frac{a^{2} m_{\nu}^{2}}{q^{2}} \frac{d \ln f^{0}}{d \ln q} \delta \rho_{\phi} .
$$

For the dark energy, we use the "fluid approach" [46] (see also [47-49]), so that the density and velocity perturbations are given by

$$
\dot{\delta}_{\phi}=\frac{3 \mathcal{H}\left(w_{\phi}-\hat{c}_{\phi}^{2}\right)\left(\delta_{\phi}+\frac{3 \mathcal{H}\left(1+w_{\phi}\right)}{1+\beta \rho_{\nu}\left(1-3 w_{\nu}\right)} \frac{\theta_{\phi}}{k^{2}}\right)-\left(1+w_{\phi}\right)\left(\theta_{\phi}+\frac{\dot{h}}{2}\right)-\left(\frac{\rho_{\nu}}{\rho_{\phi}}\right)\left[\beta \dot{\rho}_{\phi}\left(1-3 c_{\nu}^{2}\right) \delta_{\nu}+\dot{\beta} \rho_{\phi}\left(1-3 w_{\nu}\right) \delta_{\phi}\right]}{1+\beta \rho_{\nu}\left(1-3 w_{\nu}\right)},
$$

$$
\begin{aligned}
\dot{\theta}_{\phi}= & -\left[\frac{\mathcal{H}\left(1-3 \hat{c}_{\phi}^{2}\right)+\beta \rho_{\nu}\left(1-3 w_{\nu}\right) \mathcal{H}\left(1-3 w_{\phi}\right)}{1+\beta \rho_{\nu}\left(1-3 w_{\nu}\right)}\right] \theta_{\phi} \\
& +\frac{k^{2}}{1+w_{\phi}} \hat{c}_{\phi}^{2} \delta_{\phi}-\beta\left(1-3 w_{\nu}\right)\left(\frac{\rho_{\nu}}{\rho_{\phi}}\right) \\
& \times\left[\frac{k^{2}}{1+w_{\phi}} \rho_{\phi} \delta_{\phi}-\dot{\rho}_{\phi} \theta_{\phi}\right]
\end{aligned}
$$

where the dark energy anisotropic stress is assumed to be zero [50], and the sound speed $\hat{c}_{\phi}^{2}$ is defined in the frame comoving with the dark energy fluid [51]. So, in the synchronous gauge, the quantity $c_{\phi}^{2} \equiv \delta p_{\phi} / \delta \rho_{\phi}$ is related to $\hat{c}_{\phi}^{2}$ through

$$
c_{\phi}^{2} \delta_{\phi}=\hat{c}_{\phi}^{2}\left(\delta_{\phi}-\frac{\dot{\rho}_{\phi}}{\rho_{\phi}} \frac{\theta_{\phi}}{k^{2}}\right)+w_{\phi} \frac{\dot{\rho}_{\phi}}{\rho_{\phi}} \frac{\theta_{\phi}}{k^{2}} .
$$

In addition, from Eqs. (15) and (22), we have that

$$
\frac{\dot{\rho}_{\phi}}{\rho_{\phi}}=\frac{-3 \mathcal{H}\left(1+w_{\phi}\right)}{1+\beta \rho_{\nu}\left(1-3 w_{\nu}\right)} .
$$

\section{RESULTS AND DISCUSSION}

\section{A. Numerical approach}

Equipped with the background and perturbation equations, we can study this scenario by modifying the numeri- cal packages that evaluate the $\mathrm{CMB}$ anisotropies and the matter power spectrum. In particular, we modified the CAMB code [52], based on CMBFAST [53] routines. We use COSMOMC [54] in order to sample the parameter space of our model with a Markov chain Monte Carlo technique.

We assume a flat universe, with a constant equation of state dark energy fluid, cold dark matter, 2 species of massless neutrinos plus a massive one, and ten free parameters. Six of them are the standard $\Lambda$ CDM parameters, namely, the physical baryon density $\Omega_{b 0} h^{2}$, the physical cold dark matter density $\Omega_{c 0} h^{2}$, the dimensionless Hubble constant $h$, the optical depth to reionization $\tau_{\text {reion }}$, the amplitude $\left(A_{s}\right)$ and spectral index $\left(n_{s}\right)$ of primordial density fluctuations. In addition, we vary the constant dark energy equation of state parameter $w_{\phi}$ and the three parameters accounting for the neutrino mass: the present mass $m_{0}$, the logarithm of the parameter $\Delta$ related to the duration of the transition, and the logarithm of the ratio of the modulus of the mass difference over the current mass, $\log \mu$, where we define

$$
\mu \equiv \frac{\left|m_{1}-m_{0}\right|}{m_{0}} \begin{cases}\mu_{+} \equiv \frac{m_{1}}{m_{0}}-1, & m_{1}>m_{0} \\ \mu_{-} \equiv 1-\frac{m_{1}}{m_{0}}, & m_{1}<m_{0} .\end{cases}
$$

All these parameters take implicit flat priors in the regions in which they are allowed to vary (see Table I). 
TABLE I. Assumed ranges for the MaVaNs parameters.

\begin{tabular}{lc}
\hline \hline Parameter & Range \\
\hline$w_{\phi}$ & $-1<w_{\phi}<-0.5$ \\
$m_{0}$ & $0<m_{0} / \mathrm{eV}<5$ \\
$\Delta$ & $-4<\log \Delta<2$ \\
$\mu$ & $-6<\log \left(\mu_{+}\right)<0$ \\
& $-6<\log \left(\mu_{-}\right)<0$ \\
\hline \hline
\end{tabular}

Concerning the last parameter, notice that we choose to divide the parameter space between two regions: one in which the mass is decreasing over time $\left(\mu_{+}\right)$and one in which it is increasing $\left(\mu_{-}\right)$. We chose to make this separation because the impact on cosmological observables is different in each regime, as we will discuss later, and by analyzing this region separately we can gain a better insight of the physics driving the constraints in each one of them. Moreover, we do not allow for models with $w_{\phi}<$ -1 , since we are only considering scalar field models with standard kinetic terms.

For given values of all these parameters, our modified version of CAMB first integrates the background equations backward in time, in order to find the initial value of $\rho_{\phi}$ leading to the correct dark energy density today. This problem does not always admit a solution leading to well-behaved perturbations: the dark energy perturbation equations (28) and (29) become singular whenever one of the two quantities, $\rho_{\phi}$ or $\left[1+\beta \rho_{\nu}\left(1-3 w_{\nu}\right)\right]$, appearing in the denominators vanishes. As we shall see later, in the case in which the neutrino mass decreases, the background evolution is compatible with cases in which the dark energy density crosses zero, while the second term can never vanish. We exclude singular models by stopping the execution of CAMB whenever $\rho_{\phi}<0$, and giving a negligible probability to these models in COSMOMC. The physical interpretation of these pathological models will be explained in the next sections. For other models, CAMB integrates the full perturbation equations, and passes the $\mathrm{CMB}$ and matter power spectra to COSMOMC for comparison with the data.

We constrain this scenario using $\mathrm{CMB}$ data (from WMAP 5 yr [44,55], VSA [56], CBI [57], and ACBAR [58]); matter power spectrum from large-scale structure (LSS) data (2dFGRS [59] and SDSS [60]); supernovae Ia (SN) data from [61], and the HST Key project measurements of the Hubble constant [62]. ${ }^{1}$

\footnotetext{
${ }^{1}$ While this work was being finished, the SHOES (Supernova, $\mathrm{HO}$, for the Equation of State) Team [63] reduced the uncertainty on the Hubble constant by more than a factor 2 with respect to the value obtained by the HST Key Project, finding $H_{0}=74.2 \pm$ $3.6 \mathrm{~km} \mathrm{~s}^{-1} \mathrm{Mpc}^{-1}$. However, since we are taking a flat prior on $H_{0}$, and our best-fit value for $H_{0}$ is contained in their $1 \sigma$ region, we do not expect our results to be strongly affected by their results.
}

Once the posterior probability of all ten parameters has been obtained, we can marginalize over all but one or two of them, to obtain one- or two-dimensional probability distributions. We verified that the confidence limits on the usual six parameters do not differ significantly from what is obtained in the "vanilla model" [44], and therefore we only provide the results for the extra neutrino and dark energy parameters (Figs. 3, 4, 6, and 7, and Table II).

\section{B. Increasing neutrino mass}

In this model, the background evolution of the dark energy component obeys Eq. (15), which reads after division by $\rho_{\phi}$

$$
\begin{aligned}
\frac{\dot{\rho}_{\phi}}{\rho_{\phi}} & =-3 \mathcal{H}\left(1+w_{\phi}\right)-\frac{d \ln m_{\nu}}{d u} \frac{\rho_{\nu}}{\rho_{\phi}} \mathcal{H}\left(1-3 w_{\nu}\right) \\
& \equiv-\Gamma_{d}-\Gamma_{i},
\end{aligned}
$$

where the two positive quantities $\Gamma_{d}$ and $\Gamma_{i}$ represent, respectively, the dilution rate and interaction rate of the dark energy density. For any parameter choice, $\rho_{\phi}$ can only decrease with time, so that the integration of the dark energy background equation backward in time always finds well-behaved solutions with positive values of $\rho_{\phi}$. Moreover, the quantity $\left[1+\beta \rho_{\nu}\left(1-3 w_{\nu}\right)\right]$ appearing in the denominator of the dark energy perturbation equations is equal to the contribution of the dilution rate to the total energy loss rate, $\Gamma_{d} /\left(\Gamma_{d}+\Gamma_{i}\right)$. This quantity is by construction greater than zero, and the dark energy equations cannot become singular. However, when the interaction rate becomes very large with respect to the dilution rate, this denominator can become arbitrarily close to zero. Then, the dark energy perturbations can be enhanced considerably, distorting the observable spectra and conflicting the data. Actually, this amplification mechanism is well known and was studied by various authors $[20,64,65]$. It was found to affect the largest wavelengths first, and is usually referred to as the large-scale instability of coupled dark energy models. The condition for avoiding this instability can be thought to be roughly of the form

$$
\Gamma_{i}<A \Gamma_{d}
$$

where $A$ is some number depending on the cosmological parameters and on the data set (since a given data set tells how constrained the large-scale instability is, i.e. how small the denominator $\left[1+\beta \rho_{\nu}\left(1-3 w_{\nu}\right)\right]$ can be, i.e. how small should the interaction rate remain with respect to the dilution rate). The perturbations are amplified when the denominator is much smaller than 1 , so $A$ should be a number much greater than 1 . Intuitively, the condition (39) will lead to the rejection of models with small values of $\left(w_{\phi}, \Delta\right)$ and large values of $\mu_{-}$. Indeed, the interaction rate is too large when the mass variation is significant (large $\mu_{-}$) and rapid (small $\Delta$ ). The dilution rate is too small when $w_{\phi}$ is small (close to the cosmological constant 
limit). Because of that, it seems that when the dark energy equation of state is allowed to vary one can obtain a larger number of viable models if $w_{\phi}>-0.8$ early on in the cosmological evolution [66,67].

We ran COSMOMC with our full data set in order to see how much this mass-varying scenario can depart from a standard cosmological model with a fixed dark energy equation of state and massive neutrinos. In our parameter basis, this standard model corresponds to the limit $\log \mu_{-} \rightarrow-\infty$, with whatever value of $\log \Delta$. The observational signature of a neutrino mass variation during dark energy or matter domination is encoded in well-known effects, such as (i) a modification of the small-scale matter power spectrum (due to a different freestreaming history), or (ii) a change in the time of matter/radiation equality [due to a different correspondence between the values of $\left(\omega_{b}, \omega_{m}, \omega_{\nu}\right)$ today and the actual matter density at the time of equality]. On top of that, the neutrino and dark energy perturbations can approach the regime of largescale instability discussed above.

Our final results-namely, the marginalized 1D and 2D parameter probabilities-are shown in Figs. 3 and 4. The shape of the contours in $\left(\log \mu_{-}, \log \Delta\right)$ space is easily understandable with analytic approximations. The necessary condition (33) for avoiding the large-scale instability reads in terms of our model parameters

$\mu_{-}\left[\frac{1+\Delta(1+\Gamma)}{\Delta}\right]<A\left[\frac{1}{(1-\Gamma)(1-f)}\right] \frac{3 \Omega_{\phi}\left(1+w_{\phi}\right)}{\Omega_{\nu}\left(1-3 w_{\nu}\right)}$,

where we expressed the mass variation as

$$
\frac{d \ln m_{\nu}}{d u}=\left(\frac{\mu_{-}}{1-\mu_{-} \Gamma}\right)\left(\frac{1+\Delta}{\Delta}\right)(1-\Gamma)(1-f) .
$$
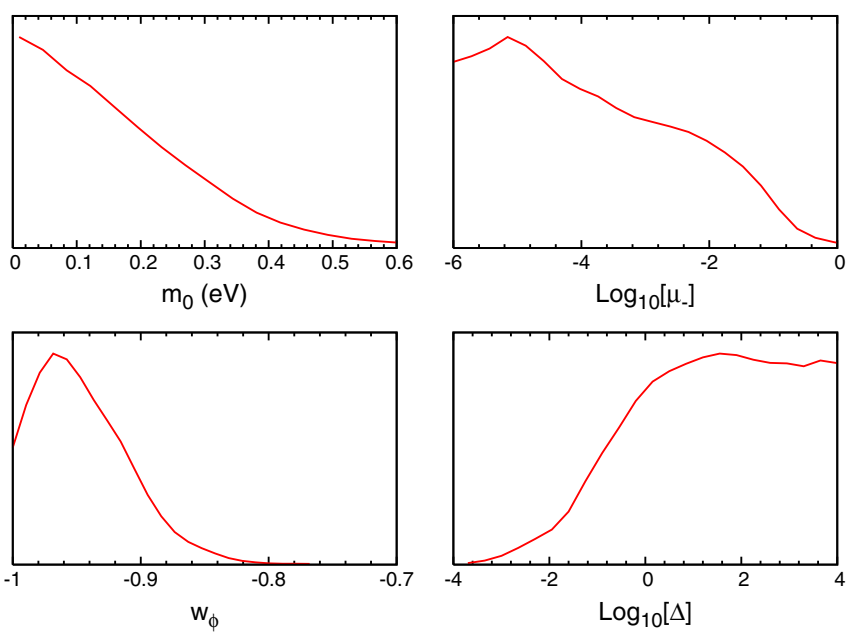

FIG. 3 (color online). Marginalized 1D probability distribution in the increasing mass case $m_{1}<m_{0}$, for the neutrino/dark energy parameters: $m_{0}, \log _{10}\left[\mu_{-}\right]$(top panels), $w_{\phi}$, and $\log \Delta$ (bottom panels).
Two limits can be clearly seen from this equation. For $\Delta \ll$ 1 (fast transitions), the upper limit on $\mu_{-}$reads

$$
\mu_{-} \lesssim A \Delta\left[\frac{1}{(1-\Gamma)(1-f)}\right] \frac{3 \Omega_{\phi}\left(1+w_{\phi}\right)}{\Omega_{\nu}\left(1-3 w_{\nu}\right)} .
$$

This corresponds to the diagonal limit in the lower half of the upper right panel of Fig. 4. In fact, the appearance of the large-scale instability is seen in models localized at the edge of the allowed region, as shown in Fig. 5.

In the opposite case of a very slow transition, $\Delta \gg 1$, it is clear from Eq. (34) that the limit on $\mu_{-}$should be independent on $\Delta$,

$$
\mu_{-} \lesssim A\left[\frac{1}{(1-\Gamma)(1-f)}\right] \frac{3 \Omega_{\phi}\left(1+w_{\phi}\right)}{\Omega_{\nu}\left(1-3 w_{\nu}\right)} .
$$

This limit corresponds to the almost vertical cut in the upper part of the plane $\left(\log \mu_{-}, \log \Delta\right)$ (upper right panel, Fig. 4).

These conditions are easier to satisfy when at the time of the transition, $\Omega_{\phi}\left(1+w_{\phi}\right)$ is large. So, in order to avoid the instability, large values of $w_{\phi}$ are preferred. However, it is well known that cosmological observables (luminosity distance relation, CMB, and LSS power spectra) better fit the data for $w$ close to -1 (cosmological constant limit). In the present model, the role of the large-scale instability is to push the best-fit value from -1 to -0.96 , but $w_{\phi}=-1$ is still allowed at the $68 \%$ C.L.

The main result of this section is that the variation of the neutrino mass is bounded to be small, not so much because of the constraining power of large-scale structure observations in the regime where neutrino freestreaming is important (i.e., small scales), but by CMB and LSS data on the largest scales, which provide limits on the possible instability in DE and neutrino perturbations.

Indeed, for the allowed models, the mass variation could be at most of order $10 \%$ for masses around $0.05 \mathrm{eV}$, and less than $1 \%$ for masses larger than $0.3 \mathrm{eV}$ : this is undetectable with small-scale clustering data, showing that the limit really comes from large scales.

With those results, we conclude that there is no evidence for a neutrino mass variation coming from the present data. In fact, as for most cosmological data analyses, the concordance $\Lambda \mathrm{CDM}$ model remains one of the best fits to the data, lying within the $68 \%$ interval of this analysis.

Nonetheless, better constraints will possibly be obtained with forthcoming data, especially the ones that probe patches of the cosmological "desert" between $z \simeq 1100$ and $z \simeq 1$, like $\mathrm{CMB}$ weak lensing [68], and/or cross correlations of different pieces of data, like CMB and galaxy-density maps [69]. We can estimate, for instance, what is the favored redshift range for the neutrino mass variation according to our results. Taking $m_{0}=0.1 \mathrm{eV}$ and the mean likelihood values for $\log \Delta$ and $\log \left[m_{1} / m_{0}\right]$, one 

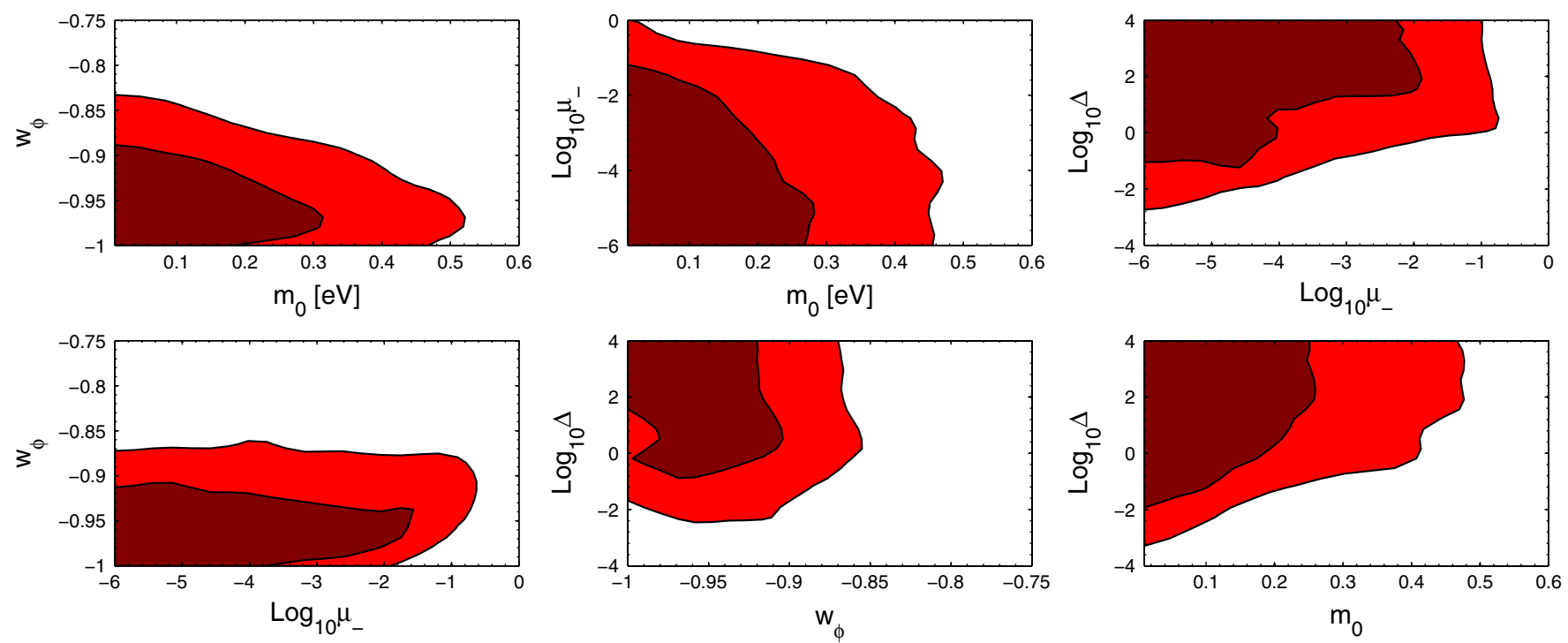

FIG. 4 (color online). Marginalized 2D probability distribution in the increasing mass case $m_{1}<m_{0}$.

can see that the bulk of the mass variation takes place around $z \sim 20$, a redshift that possibly will be probed by future tomographic probes like weak lensing [70,71] and especially $21 \mathrm{~cm}$ absorption lines [72-75]. Those will help not only to disentangle some degeneracies in the parameter space, but will also allow for direct probes of the neutrino mass in different redshift slices.

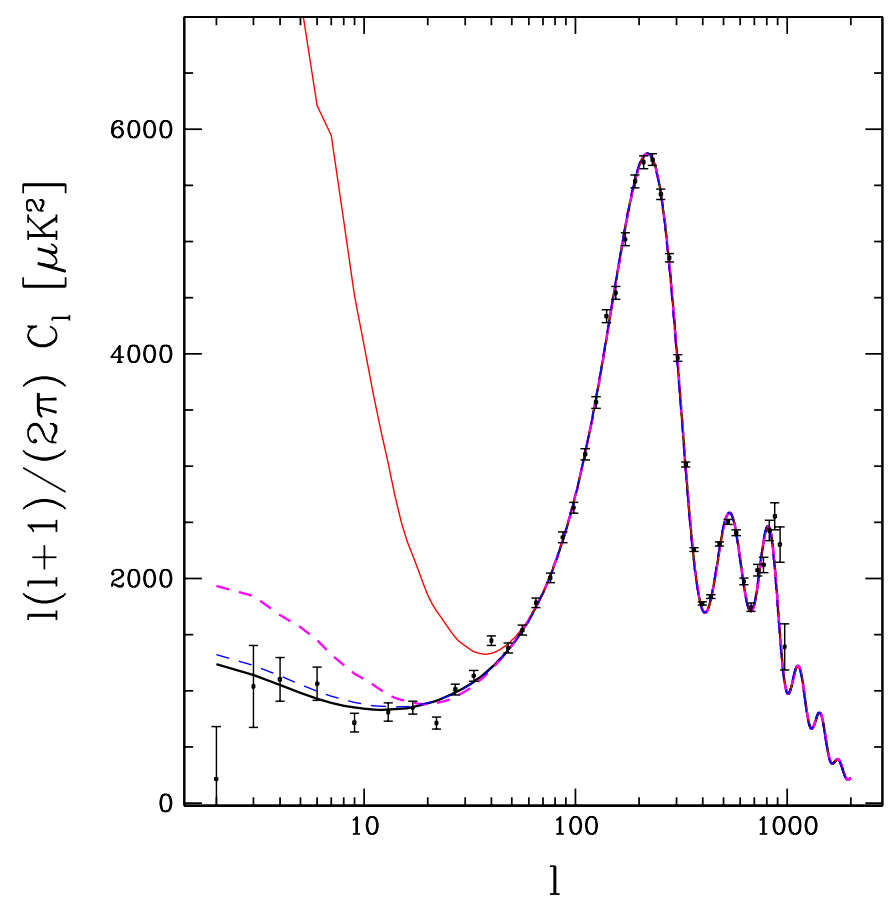

\section{Decreasing neutrino mass}

In this case, the evolution rate of the dark energy density is still given by Eq. (32) but with an opposite sign for the interaction rate: it can be summarized as

$$
\frac{\dot{\rho}_{\phi}}{\rho_{\phi}}=-\Gamma_{d}+\Gamma_{i}
$$

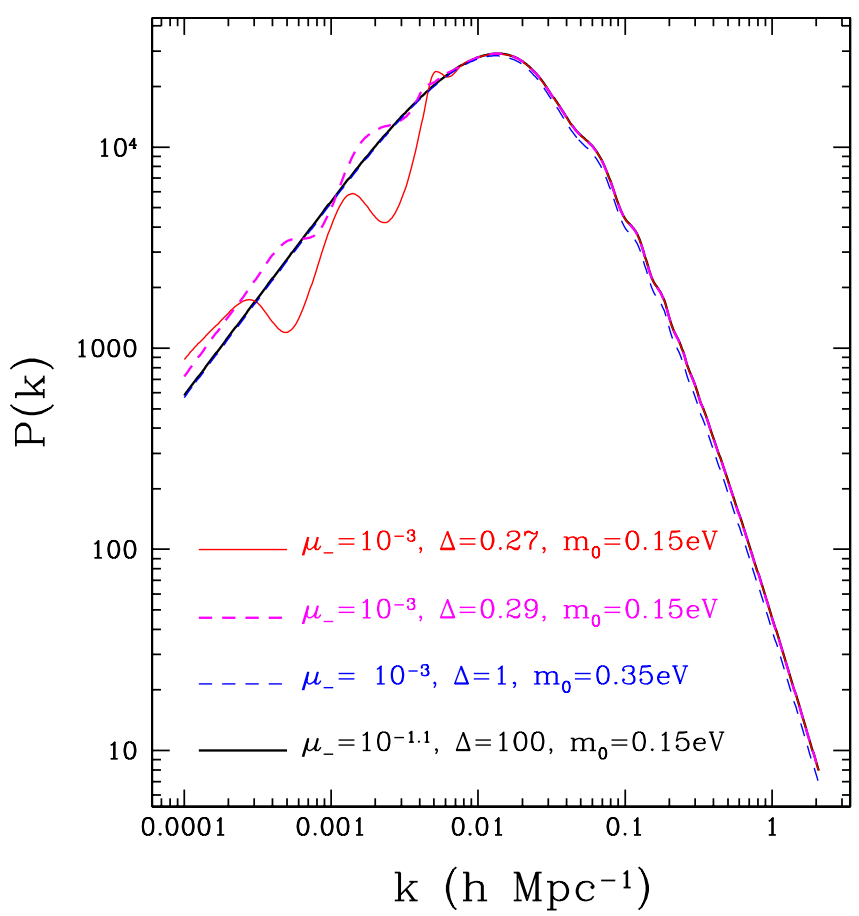

FIG. 5 (color online). CMB anisotropies and matter power spectra for some mass-varying models with increasing mass, showing the development of the large-scale instability. The cosmological parameters are set to our best-fit values, except for the ones shown in the plot. The data points in the CMB spectrum correspond to the binned WMAP 5 yr data. 

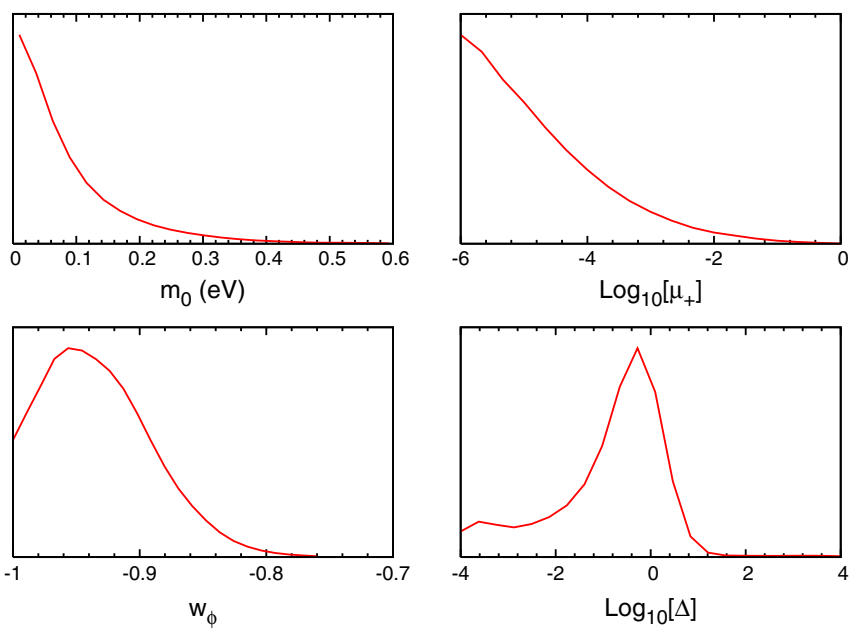

FIG. 6 (color online). Marginalized 1D probability distribution (red/solid lines) for the decreasing mass case $m_{1}>m_{0}$, for neutrino/dark energy parameters: $m_{0}, \log \left[\mu_{+}\right]$(top panels), $w_{\phi}$, and $\log \Delta$ (bottom panels).

with $\Gamma_{d}$ and $\Gamma_{i}$ both positive. In principle, the interaction rate could overcome the dilution rate, leading to an increase of $\rho_{\phi}$. Hence, the integration of the dark energy evolution equation backward in time can lead to negative values of $\rho_{\phi}$, and the prior $\rho_{\phi}>0$ implemented in our CAMB version is relevant. Still, the denominator $[1+$ $\left.\beta \rho_{\nu}\left(1-3 w_{\nu}\right)\right]$ can never vanish since it is equal to $\Gamma_{d} /\left(\Gamma_{d}-\Gamma_{i}\right)$.

Well before the transition, the interaction rate is negligible and $\dot{\rho}_{\phi}$ is always negative. We conclude that $\beta=$ $d \ln m_{\nu} / d \rho_{\phi}$ starts from small positive values and increases. If the condition

$$
\Gamma_{i}<\Gamma_{d}
$$

is violated during the transition, $\dot{\rho}_{\phi}$ will cross zero and become positive. This corresponds to $\beta$ growing from zero to $+\infty$, and from $-\infty$ to some finite negative value. After $\Gamma_{i} / \Gamma_{d}$ has reached its maximum, $\beta$ undergoes the opposite evolution. Reaching $\rho_{\phi}=0$ is only possible if $\rho_{\phi}$ has a nonmonotonic evolution, i.e. if (39) is violated. However, the perturbations diverge even before reaching this singular point: when $\beta$ tends to infinity, it is clear from Eq. (26) that the neutrino perturbation derivatives become arbitrarily large. We conclude that in this model, the condition (39) is a necessary condition for avoiding instabilities, but not a sufficient condition: the data are expected to put a limit on the largest possible value of $\beta$, which will always be reached before $\dot{\rho}_{\phi}$ changes sign, i.e. before the inequality (39) is saturated. Hence, the condition for avoiding the instability is intuitively of the form of (33), but now with $A$ being a number smaller than 1 .

We then ran COSMOMC with the full data set and obtained the marginalized $1 \mathrm{D}$ and $2 \mathrm{D}$ parameter probabilities shown in Figs. 6 and 7. The major differences with respect to the increasing mass case are a stronger bound on $m_{0}$, a much stronger bound on $\mu_{-}$, and the fact that large values of $\Delta$ are now excluded. This can be understood as follows. In order to avoid instabilities, it is necessary to satisfy the inequalities (36) and (37), but with a much smaller value of $A$ than in the increasing mass case; hence, the contours should look qualitatively similar to those obtained previously, but with stronger bounds. This turns out to be the case, although in addition, large $\Delta$ values are now excluded. Looking at the mass variation for large $\Delta$ in Fig. 1, we see that in this limit the energy transfer takes place essentially at low redshift. Hence, the interaction rate is large close to $z=0$. In many models, this leads to positive values of $\dot{\rho}_{\phi}$ at the present time, to a nonmonotonic behavior of the dark energy density, and to diverging
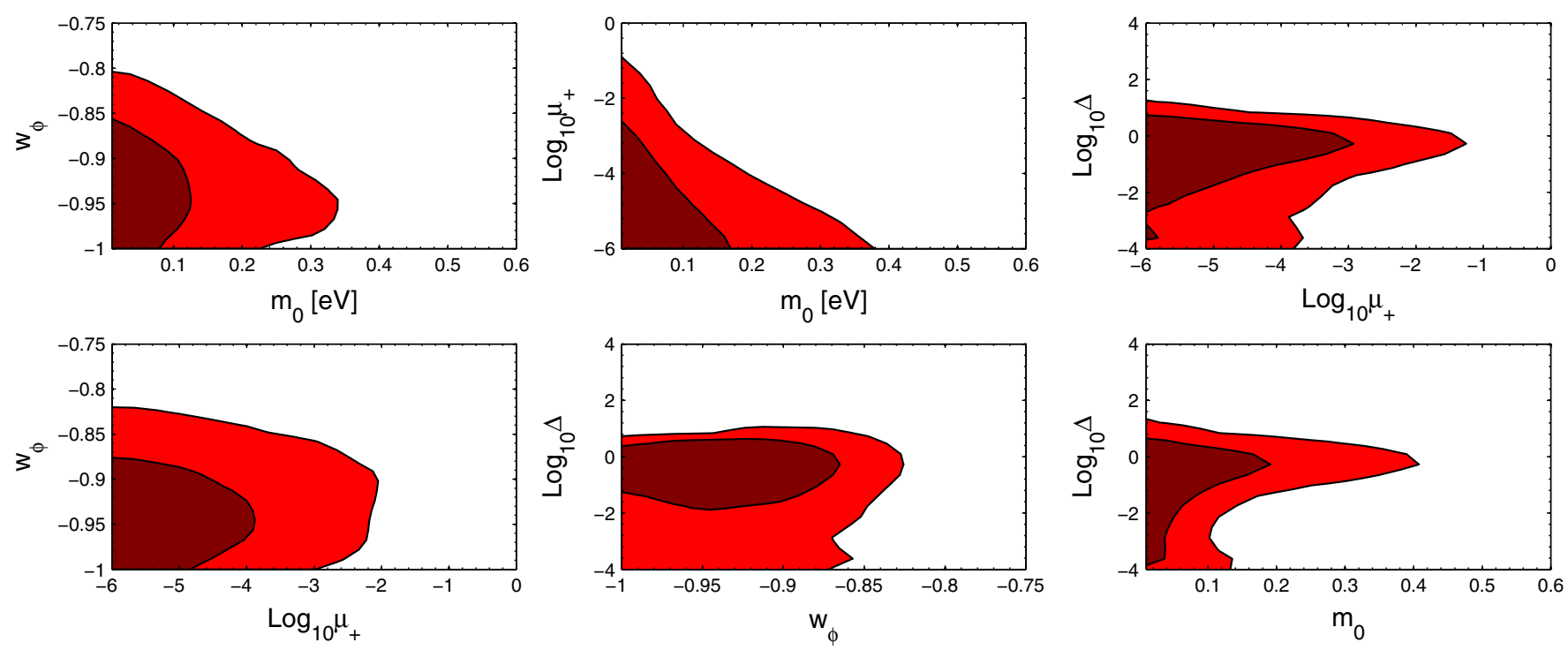

FIG. 7 (color online). Marginalized 2D probability distribution for decreasing mass, $m_{1}>m_{0}$. 
TABLE II. Results for increasing and decreasing neutrino mass, using WMAP 5 yr + small scale CMB + LSS + SN + HST data.

\begin{tabular}{lcc}
\hline \hline & $(+)$ region $95 \%(68 \%)$ C.L. & $(-)$ region 95\% (68\%) C.L. \\
\hline$w_{\phi}$ & $<-0.85(<-0.91)$ & $<-0.87(<-0.93)$ \\
$m_{0}(\mathrm{eV})$ & $<0.28(<0.10)$ & $<0.43(<0.21)$ \\
$\log \mu_{+}$ & $<-2.7(<-4.5)$ & $\ldots$ \\
$\log \mu_{-}$ & $\ldots$ & $<-1.3(<-3.1)$ \\
$\log \Delta$ & {$[-3.84 ; 0.53]([-2.20 ; 0.05])$} & {$[-0.13 ; 4]([0.56 ; 4])$} \\
\hline \hline
\end{tabular}

perturbations. This can only be avoided when $w$ is large with respect to -1 , i.e. when the dilution rate is enhanced. Hence, in this model, the need to avoid diverging perturbations imposes a strong parameter correlation between $w$ and $\Delta$. However, values of $w$ greater than -0.8 are not compatible with the supernovae, CMB, and LSS data set; this slices out all models with large $\Delta$.

The fact that the bound on $m_{0}$ is stronger in the decreasing mass case is also easily understandable: for the same value of the mass difference $\mu_{ \pm}=\left|m_{1}-m_{0}\right| / m_{0}$, a given $m_{0}$ corresponds to a larger mass $m_{1}$ in the decreasing mass case. It is well known that CMB and LSS data constrain the neutrinos mass through its background effect, i.e. through its impact on the time of matter/radiation equality for a given dark matter abundance today. The impact is greater when $m_{1}$ is larger, i.e. in the decreasing mass case; therefore, the bounds on $m_{0}$ are stronger.

\section{CONCLUDING REMARKS}

In this work we analyzed some mass-varying neutrino scenarios in a nearly model independent way, using a general and well-behaved parametrization for the neutrino mass, including variations in the dark energy density in a self-consistent way, and taking neutrino/dark energy perturbations into account.

Our results for the background, CMB anisotropies, and matter power spectra are in agreement with previous analyses of particular scalar field models, showing that the results obtained with this parametrization are robust and encompass the main features of the MaVaNs scenario.

Moreover, a comparison with cosmological data shows that only small mass variations are allowed, and that MaVaNs scenarios are mildly disfavored with respect to the constant mass case, especially when neutrinos become lighter as the Universe expands. In both cases, neutrinos can change significantly the evolution of the dark energy density, leading to instabilities in the dark energy and/or neutrino perturbations when the transfer of energy between the two components per unit of time is too large. These instabilities can only be avoided when the mass varies by a very small amount, especially in the case of a decreasing neutrino mass. Even in the case of increasing mass, better constraining the model with forthcoming data will be a difficult task, since it mimics a massless neutrino scenario for most of the cosmological time.

One should keep in mind that our analysis assumes a constant equation of state for dark energy and a monotonic behavior for the mass variation. Even though those features are present in most of the simplest possible models, more complicated models surely can evade the constraints we obtained in our analysis.

Finally, those constraints will improve with forthcoming tomographic data. If any of the future probes indicate a mismatch in the values of the neutrino mass at different redshifts, we could arguably have a case made for the mass-varying models.

\section{ACKNOWLEDGMENTS}

We would like to thank Luca Amendola, Alberto Fernández-Soto, Gennaro Miele, Miguel Quartin, Rogerio Rosenfeld, and José W.F. Valle for discussions concerning an earlier version of this work. This work was supported by the European Union (Contracts No. RII3-CT2004-506222 and No. MRTN-CT-2004-503369, and Marie Curie Training Network "UniversetNet" No. MRTN-CT2006-035863), by the Spanish Grants No. FPA2008-00319 (MEC) and No. PROMETEO/2009/091 (Generalitat Valenciana), and by a MEC-IN2P3 agreement. U.F. is supported by an I3P-CSIC grant. This work made some progress during a fruitful stay at the Galileo Galilei Institute for Theoretical Physics, supported by INFN. We also acknowledge the use of the Legacy Archive for Microwave Background Data Analysis (LAMBDA). Support for LAMBDA is provided by the NASA Office of Space Science. 
[1] A. G. Riess et al. (Supernova Search Team Collaboration), Astron. J. 116, 1009 (1998).

[2] S. Perlmutter et al. (Supernova Cosmology Project Collaboration), Astrophys. J. 517, 565 (1999).

[3] J. Frieman, M. Turner, and D. Huterer, Annu. Rev. Astron. Astrophys. 46, 385 (2008).

[4] E. J. Copeland, M. Sami, and S. Tsujikawa, Int. J. Mod. Phys. D 15, 1753 (2006).

[5] P. J.E. Peebles and B. Ratra, Rev. Mod. Phys. 75, 559 (2003).

[6] R. R. Caldwell and M. Kamionkowski, arXiv:0903.0866 [Annu. Rev. Nucl. Part. Sci. (to be published)].

[7] R. R. Caldwell, R. Dave, and P. J. Steinhardt, Phys. Rev. Lett. 80, 1582 (1998).

[8] E. V. Linder, Rep. Prog. Phys. 71, 056901 (2008).

[9] A. Albrecht et al., arXiv:astro-ph/0609591.

[10] P. Gu, X. Wang, and X. Zhang, Phys. Rev. D 68, 087301 (2003).

[11] R. Fardon, A. E. Nelson, and N. Weiner, J. Cosmol. Astropart. Phys. 10 (2004) 005.

[12] R. D. Peccei, Phys. Rev. D 71, 023527 (2005).

[13] L. Amendola, M. Baldi, and C. Wetterich, Phys. Rev. D 78, 023015 (2008).

[14] C. Wetterich, Phys. Lett. B 655, 201 (2007).

[15] S. Hannestad, Annu. Rev. Nucl. Part. Sci. 56, 137 (2006).

[16] J. Lesgourgues and S. Pastor, Phys. Rep. 429, 307 (2006).

[17] N. Afshordi, M. Zaldarriaga, and K. Kohri, Phys. Rev. D 72, 065024 (2005).

[18] O.E. Bjaelde, A.W. Brookfield, C. van de Bruck, S. Hannestad, D. F. Mota, L. Schrempp, and D. TocchiniValentini, J. Cosmol. Astropart. Phys. 01 (2008) 026.

[19] R. Bean, E. E. Flanagan, and M. Trodden, New J. Phys. 10, 033006 (2008).

[20] R. Bean, E. E. Flanagan, and M. Trodden, Phys. Rev. D 78, 023009 (2008).

[21] R. Bean, E. E. Flanagan, I. Laszlo, and M. Trodden, Phys. Rev. D 78, 123514 (2008).

[22] A. E. Bernardini and O. Bertolami, Phys. Lett. B 662, 97 (2008).

[23] A. W. Brookfield, C. van de Bruck, D.F. Mota, and D. Tocchini-Valentini, Phys. Rev. Lett. 96, 061301 (2006).

[24] A. W. Brookfield, C. van de Bruck, D. F. Mota, and D. Tocchini-Valentini, Phys. Rev. D 73, 083515 (2006); 76, 049901(E) (2007).

[25] K. Ichiki and Y. Y. Keum, J. Cosmol. Astropart. Phys. 06 (2008) 005.

[26] G. B. Zhao, J.Q. Xia, and X.M. Zhang, J. Cosmol. Astropart. Phys. 07 (2007) 010.

[27] G.W. Anderson and S.M. Carroll, arXiv:astro-ph/ 9711288.

[28] L. Amendola, Phys. Rev. D 62, 043511 (2000).

[29] L. Amendola and D. Tocchini-Valentini, Phys. Rev. D 64, 043509 (2001).

[30] G. R. Farrar and P. J.E. Peebles, Astrophys. J. 604, 1 (2004).

[31] U. França and R. Rosenfeld, Phys. Rev. D 69, 063517 (2004).

[32] G. Huey and B.D. Wandelt, Phys. Rev. D 74, 023519 (2006).

[33] S. Das, P. S. Corasaniti, and J. Khoury, Phys. Rev. D 73, 083509 (2006).
[34] M. Quartin, M. O. Calvao, S. E. Joras, R. R. R. Reis, and I. Waga, J. Cosmol. Astropart. Phys. 05 (2008) 007.

[35] G. La Vacca, J. R. Kristiansen, L. P. L. Colombo, R. Mainini, and S.A. Bonometto, J. Cosmol. Astropart. Phys. 04 (2009) 007.

[36] M. Chevallier and D. Polarski, Int. J. Mod. Phys. D 10, 213 (2001).

[37] E. V. Linder, Phys. Rev. Lett. 90, 091301 (2003).

[38] G. Mangano, G. Miele, S. Pastor, T. Pinto, O. Pisanti, and P. D. Serpico, Nucl. Phys. B729, 221 (2005).

[39] R. Rosenfeld, Phys. Rev. D 75, 083509 (2007).

[40] P. Brax, C. van de Bruck, A. C. Davis, J. Khoury, and A. Weltman, Phys. Rev. D 70, 123518 (2004).

[41] P. S. Corasaniti and E. J. Copeland, Phys. Rev. D 67, 063521 (2003).

[42] M. Douspis, Y. Zolnierowski, A. Blanchard, and A. Riazuelo, Astron. Astrophys. 488, 47 (2008).

[43] S. Linden and J. M. Virey, Phys. Rev. D 78, 023526 (2008).

[44] E. Komatsu et al. (WMAP Collaboration), Astrophys. J. Suppl. Ser. 180, 330 (2009).

[45] C. P. Ma and E. Bertschinger, Astrophys. J. 455, 7 (1995).

[46] W. Hu, Astrophys. J. 506, 485 (1998).

[47] R. Bean and O. Dore, Phys. Rev. D 69, 083503 (2004).

[48] S. Hannestad, Phys. Rev. D 71, 103519 (2005).

[49] T. Koivisto and D.F. Mota, Phys. Rev. D 73, 083502 (2006).

[50] D.F. Mota, J. R. Kristiansen, T. Koivisto, and N.E. Groeneboom, Mon. Not. R. Astron. Soc. 382, 793 (2007).

[51] J. Weller and A. M. Lewis, Mon. Not. R. Astron. Soc. 346, 987 (2003).

[52] A. Lewis, A. Challinor, and A. Lasenby, Astrophys. J. 538, 473 (2000); http://camb.info/.

[53] U. Seljak and M. Zaldarriaga, Astrophys. J. 469, 437 (1996); http://cfa-www.harvard.edu/ mzaldarr/ CMBFAST/cmbfast.html.

[54] A. Lewis and S. Bridle, Phys. Rev. D 66, 103511 (2002); http://cosmologist.info/cosmomc/.

[55] J. Dunkley et al. (WMAP Collaboration), Astrophys. J. Suppl. Ser. 180, 306 (2009).

[56] P.F. Scott et al., Mon. Not. R. Astron. Soc. 341, 1076 (2003).

[57] T. J. Pearson et al., Astrophys. J. 591, 556 (2003).

[58] C. 1. Kuo et al. (ACBAR Collaboration), Astrophys. J. 600, 32 (2004).

[59] S. Cole et al. (2dFGRS Collaboration), Mon. Not. R. Astron. Soc. 362, 505 (2005).

[60] M. Tegmark et al. (SDSS Collaboration), Phys. Rev. D 74, 123507 (2006).

[61] M. Kowalski et al. (Supernova Cosmology Project Collaboration), Astrophys. J. 686, 749 (2008).

[62] W. L. Freedman et al., Astrophys. J. 553, 47 (2001).

[63] A. G. Riess et al., Astrophys. J. 699, 539 (2009).

[64] J. Valiviita, E. Majerotto, and R. Maartens, J. Cosmol. Astropart. Phys. 07 (2008) 020.

[65] M. B. Gavela, D. Hernandez, L. L. Honorez, O. Mena, and S. Rigolin, J. Cosmol. Astropart. Phys. 07 (2009) 034.

[66] J. Valiviita, R. Maartens, and E. Majerotto, arXiv:0907.4987.

[67] E. Majerotto, J. Valiviita, and R. Maartens, arXiv:0907.4981. 
[68] J. Lesgourgues, L. Perotto, S. Pastor, and M. Piat, Phys. Rev. D 73, 045021 (2006).

[69] J. Lesgourgues, W. Valkenburg, and E. Gaztañaga, Phys. Rev. D 77, 063505 (2008).

[70] S. Hannestad, H. Tu, and Y. Y. Y. Wong, J. Cosmol. Astropart. Phys. 06 (2006) 025.

[71] T. D. Kitching, A. F. Heavens, L. Verde, P. Serra, and A. Melchiorri, Phys. Rev. D 77, 103008 (2008).
[72] A. Loeb and M. Zaldarriaga, Phys. Rev. Lett. 92, 211301 (2004).

[73] A. Loeb and J.S. Wyithe, Phys. Rev. Lett. 100, 161301 (2008).

[74] Y. Mao, M. Tegmark, M. McQuinn, M. Zaldarriaga, and O. Zahn, Phys. Rev. D 78, 023529 (2008).

[75] J. R. Pritchard and E. Pierpaoli, Phys. Rev. D 78, 065009 (2008). 